Carlo Alberto Scirè: None declared, Alessia Alunno: None declared, Xenofon Baraliakos Grant/research support from: Grant/research support from: AbbVie, BMS, Celgene, Chugai, Merck, Novartis, Pfizer, UCB and Werfen, Consultant of: AbbVie, BMS, Celgene, Chugai, Merck, Novartis, Pfizer, UCB and Werfen, Speakers bureau: AbbVie, BMS, Celgene, Chugai, Merck, Novartis, Pfizer, UCB and Werfen, Christian Dejaco: None declared DOI: 10.1136/annrheumdis-2020-eular.6070

\section{AB1084 $\quad$ A SYSTEMATIC REVIEW OF THE ABILITY OF WHOLE BODY MRI TO ASSESS DISEASE ACTIVITY AND TREATMENT RESPONSE IN INFLAMMATORY ARTHRITIS}

V. Choida ${ }^{1,2}$, A. V. Madenidou ${ }^{1,2}$, D. Sen ${ }^{1,2}$, M. Hall-Craggs ${ }^{3,4}$, C. Ciurtin ${ }^{1,2}$ ${ }^{1}$ University College London, Centre for Adolescent Rheumatology Versus Arthritis, London, United Kingdom; ${ }^{2}$ University College London Hospitals NHS Foundation Trust, Rheumatology Department, London, United Kingdom; ${ }^{3}$ University College London Hospitals NHS Foundation Trust, Radiology Department, London, United Kingdom; ${ }^{4}$ University College London, Centre for Medical Imaging, London, United Kingdom

Background: Whole body MRI (WBMRI) is an imaging technique that allows the assessment of the spine and peripheral joints in patients with inflammatory arthritis (IA) in a single examination. Depending on the protocol, it can potentially identify synovitis, enthesitis, spondyloarthritis and chronic structural changes. Objectives: To evaluate the performance of WBMRI in patients with IA for detecting inflammation compared with clinical assessments and to show changes in response to treatment.

Methods: We conducted a systematic search of the electronic databases MEDLINE, EMBASE and Cochrane Library. Two authors selected independently the eligible studies, extracted the predefined data and assessed the quality using the QUADAS2 tool. Studies that reported a)disease activity scores, b)patient or physician reported outcomes or c)results from other imaging tests in IA patients who underwent WBMRI were included.

Results: Fourteen studies out of $\mathbf{4 7 1}$ met our inclusion criteria. The majority of the studies were performed in Spondyloarthritis [SpA] $(n=9)$, followed by Rheumatoid Arthritis [RA] $(n=4)$ and Psoriatic Arthritis [PsA] patients $(n=3)$. Nine studies provided clinical and MRI outcome measures. There was great heterogeneity in the quality of studies, disease specific outcomes reported and methodology used to compare with MRI findings. One study documented low correlation between 28 swollen/tender joint count and MRI bone marrow oedema (BME)/ synovitis in RA patients, whereas another reported that $31 \%$ of $M R I$ negative joints (other than hand joints) exhibited tenderness. In PsA, one study demonstrated correlation between 28 swollen joint count and BME ( $r=0.54, p=0.03)$. Superiority of WBMRI in the detection of synovitis and enthesitis over clinical examination was documented in two studies with SpA patients. A third study in SpA showed a ranging agreement of 49 to $100 \%$ between clinical and WBMRI enthesitis.

Treatment response to biologics was assessed by WBMRI in 7 studies ( 5 in SpA, 2 in RA). In RA, one study showed numerical (but not statistically significant) reduction of WBMRI joint count at week 16 and 52 of Adalimumab treatment, whereas the reduction was statistically significant for the subset of patients achieving good EULAR response at week 16 . The other study demonstrated a reduction in WBMRI synovitis and bone oedema scores after 1 year of anti-TNF or Tocilizumab treatment (median DAS28 score decreased from 5.1 to 2.1). A multicentre open label study reported a reduction in the number of MRI enthesitis lesions, spinal and sacroiliac joint scores at week 48, year 2 and 3 of Etanercept treatment in SpA patients. The mean BASDAI score decreased from 5.4 at baseline to 1.5 at year 2 and 2.2 at year 3. Improvement in WBMRI scores in SpA was also documented in one Adalimumab and one further Etanercept study.

Conclusion: There was a variable level of correlation between clinical and WBMRI outcome measures across the included studies. The clinical significance of inflammation detected by WBMRI in some studies remains unclear. Many of the devised WBMRI scores appear to decrease after biologic treatment. Further studies are needed to determine the accuracy of WBMRI in detecting inflammation and its potential utility for clinical practice.

Disclosure of Interests: Varvara Choida: None declared, Anastasia-Vasiliki Madenidou: None declared, Debajit Sen: None declared, Margaret Hall-Craggs: None declared, Coziana Ciurtin Grant/research support from: Pfizer, Consultant of: Roche, Modern Biosciences

DOI: 10.1136/annrheumdis-2020-eular.2576

\section{AB1085 \\ ASSESSMENT OF DIAGNOSTIC DELAY IN PATIENTS AFFECTED BY ENTEROPATHIC SPONDYLOARTHRITIS: A CROSS-SECTIONAL STUDY}

P. Conigliaro $^{1}$, M. S. Chimenti ${ }^{1}$, A. D'antonio ${ }^{1}$, A. Wlderk ${ }^{2}$, L. Sichi ${ }^{1}$,

P. Triggianese ${ }^{1}$, F. Sabuzi ${ }^{2}$, G. Sena ${ }^{3}$, N. Alfieri ${ }^{3}$, V. Da Ros ${ }^{2}$, L. Biancone ${ }^{3}$,
R. Perricone ${ }^{1} .{ }^{1}$ University of Rome Tor Vergata, Rheumatology, Allergology and Clinical Immunology, Department of "Medicina dei Sistemi", Rome, Italy; ${ }^{2}$ University of Rome Tor Vergata, Department of Diagnostic Imaging and Interventional Radiology, Rome, Italy; ${ }^{3}$ University of Rome Tor Vergata, Gastroenterology Unit, Department of “Medicina dei Sistemi", Rome, Italy

Background: Diagnostic delay of spondyloarthritis ( $\mathrm{SpA}$ ) has been established even in combination with inflammatory bowel disease and may contribute to radiographic progression and disability.

Objectives: We aimed to evaluate diagnostic delay in enteropathic SpA (eSpA) and explore associated demographic, clinical, and radiographic characteristics. Methods: We analysed consecutive eSpA patients referred to the combined gastro-rheumatologic clinic of the University of Rome Tor Vergata. Diagnostic delay was defined as the time interval from the date of first symptoms to the date of diagnosis. Conventional radiography $(\mathrm{CR})$ and magnetic resonance images (MRI) of sacroiliac (SI) joints and spine were performed in axial (ax)SpA patient and examined by two independent radiologists. MRI were assessed for the presence of active/chronic inflammatory lesions, disease activity by ASDAS and inflammatory markers. Statistical analyses were performed using Mann-Whitney, chi square/Fisher tests and covariance analysis (SPSS software).

Results: 190 eSpA patients (124 female, mean age $47.5 \pm 12.8$ years, disease duration $72 \pm 67.4$ months, 73 UC/117 CD; 118 peripheral SpA, 72 axSpA including 44 non radiographic ( $n r)$-axSpA) were evaluated. Axial eSpA patients had a higher prevalence of men sex $(p<0.0001)$, HLA-B27 positivity $(p=0.004)$, uveitis $(p=0.01)$ and pancolitis $(p<0.006)$ compared with peripheral eSpA. AxeSpA patients displayed higher ESR, ASDAS, and VAS pain compared with peripheral ESpA ( $p=0.0006, p=0.001, p=0.019$, respectively). A higher prevalence of csDMARDs was detected in peripheral eSpA compared with axSpA $(p=0.002)$ while treatment with cs and bDMARDs was similar in rad-axSpA and nr-axSpA patients.

Median diagnostic delay in eSpA was 48 months (IQR 6-77) with no difference between axial and peripheral patients. Rad-ax-SpA patients displayed a higher diagnostic delay compared with nr-axSpA (median/IQR 36/17-129 vs 31/10-57 months, $p=0.03$ ). Patients with rad-axSpA were older and with longer disease duration than patients with $n r-a x \operatorname{SpA}(p=0.005$ and $p=0.019)$. Low education status and high rate of employment were found in rad-axSpA compared with nr-ax$\operatorname{SpA}(p=0.003$ and $p=0.03$, respectively).

Rad-axSpA patients with sclerosis, syndesmophytes and bridge at CR had a higher diagnostic delay than those without lesions $(p=0.03, p=0.043, p<0.0001$ Fig. $1 \mathrm{~A}-\mathrm{C}$ ). Men showed a higher prevalence of spine damage lesions than women as sclerosis $(p=0.02)$, squaring $(p=0.0006)$, syndesmophytes $(p=0.0028)$ and bridges $(p=0.007)$. Longer disease duration was detected in patients with radiographic damage as bridge $(p<0.0001)$ and sacroiliitis grade $3(p=0.04)$. On $\mathrm{MRI}, \mathrm{SI}$ bone oedema was associated with reduced diagnostic delay $(p=0.04)$ while bone erosions was associated with higher diagnostic delay $(p=0.002)$ compared with that in patients without these lesions (Fig. 1D-E). Rad-axSpA women had a higher prevalence of $S I$ damage lesions at MRI than men $(p=0.001)$ Patients with psoriasis displayed a higher diagnostic delay compared to those without skin involvement $(p=0.004)$.
A

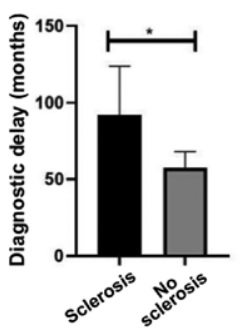

D

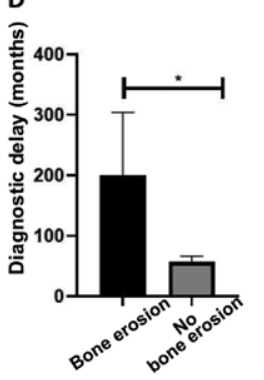

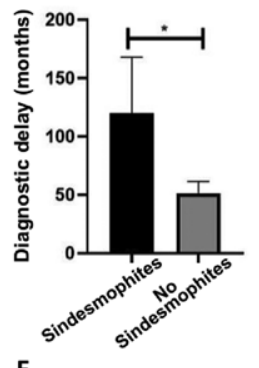

E

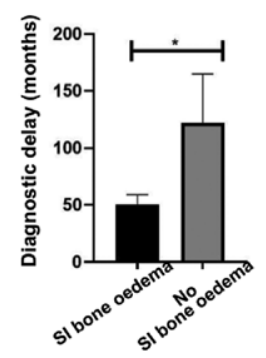

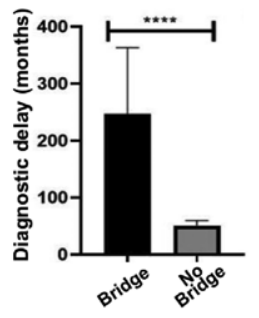

Figure 1. 